\title{
A New Lightning Protection Method for Distribution Transformers
}

\author{
SHI Xunjie ${ }^{1, a}$, LÜ Fangcheng ${ }^{1, b}$, DING Yujian ${ }^{1,2, c}$ \\ ${ }^{1}$ School of Electrical and Electronic Engineering, North China Electric Power University, Changping \\ District, Beijing 102206 China \\ ${ }^{2}$ China Electric Power Research Institute, Haidian District, Beijing 100192 China \\ axunjie_shi@163.com, 'blfc@ncepu.edu.cn, 'dingyj@epri.sgcc.com.cn
}

Keywords: over-voltage, electromagnetic simulation, distribution transformer, lightning protection

\begin{abstract}
The distribution transformers are easily damaged when struck by lightning, the reliability of power supply and distribution system as well as the operational safety will be affected seriously. Therefore, it is necessary to find out a relatively thorough lightning protection measure, to ensure the safe and stable opreation of distribution transformer. This paper puts forward a new protection method which combines surge arresters with power cables. And then, Electro-Magnetic Transient Program (EMTP) is used to verify the effectiveness of the method. The results shows that the method is simple and easy to implement, without additional electrical equipment, provides a new effective theoretical way for lightning protection of distribution transformers.
\end{abstract}

\section{Introduction}

The $10 \mathrm{kV}$ (or $35 \mathrm{kV}$ ) distribution network is an important part of the power system, and is the main power supply network in rural areas and suburbs. The reliable and stable power supply is a critical guarantee for the economic development of the rural areas and suburbs. However, the overhead distribution lines in suburbs and rural areas usually go through extensive open areas, are susceptible to be struck by lightning. As the lightning wave passes along the lines to distribution transformers, it will be damaged easily, threatening the reliability and the operational safety of power distribution system. The main reason for the broken of the transformers is that the protection method is inappropriate.

When power transformers are struck by lightning, complicated electromagnetic oscillation process will appear in the internal windings, making high overvoltage occur between each point on the windings, such as inter-turn, inter-layer and inter-reel. Reducing the amplitudes and gradients of the intrusion impulse voltage has a very important significance for the protection of insulation of winding, especially the vertical insulation.The proposed method can prevent the hazards of the positive transform overvoltages and inverse transform overvoltage, and can also reduce the amplitudes and gradients of the invading voltage of the high-voltage side, thereby protecting the insulation of windings.

\section{A New Protection Method for Distribution Transformers}

The simplified wiring circuit is as shown in fig. 1, the connecting conductors between the arresters and transformers are single-core power cables.

The length of the cables is better to take $15 \sim 50 \mathrm{~m}$, exceeding $100 \mathrm{~m}$ would be improper. If the length is too long, it would not only increase the investment but also accelerate the aging the cable due to the induced voltage and current of the outer layers when in normal operation. Braid copper should be used as shield, its flows are relatively greater than copperbelts, has better heat dissipation, meanwhile it can also prevent the occurrence of electromagnetic squeeze effectively ${ }^{[1]}$. The shields and the armors should be connected with the ground port of the arresters at the head of the cables, and at the end of the cables the shields and the armors should be connected together only. When lightning current flows through the cores, corresponding currents will be induced in the shields and armors, and 
the produced electromagnetic blockade effect will make the lightning currents that flow through the cores decrease greatly ${ }^{[2]}$.

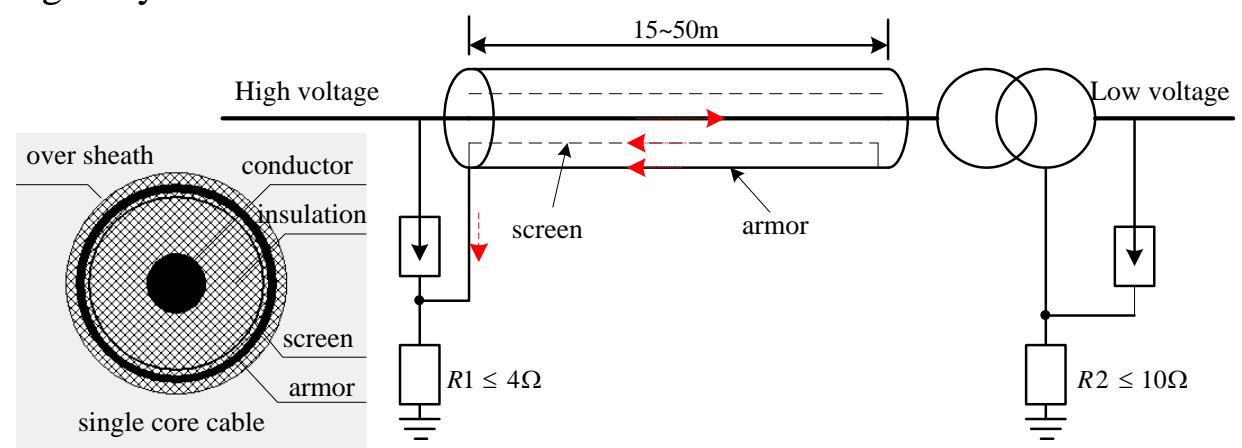

Fig.1 Simplified wiring diagram

The power frequency resistance of grounding body 1 should be less than $4 \Omega$, and the resistance of grounding body 2 can be slightly larger, but no more than $10 \Omega$. The distance between the two grounding bodys should be bigger than $5 \mathrm{~m}$.

These not long cables is somehow equivalent to parallel capacitances, which can reduce the gradients of the invading voltage, and is conducive to the discharge of the arresters ${ }^{[3]}$.

\section{Multiple Catadioptric process of Traveling Wave in The Line}

A line, whose wave impedance is $z l$ and length is $l$, is connected between line 1 and line 2, as shown in fig. 2. Assuming the length of line 1 and line 2 is infinite, or a more general view is that the reflected waves generated in distance have not yet reached point 1 and 2.

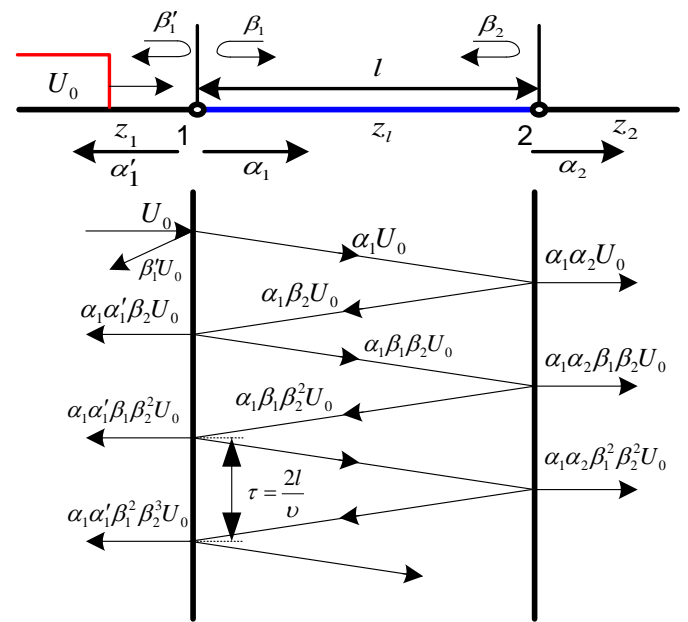

Fig. 2 Multiple catadioptric process of traveling wave in the line

Assuming there is an infinite cartesian wave $U_{0}$ traveling from line 1 to line 2, then the wave will have multiple catadioptric process between piont 1 and piont 2 . When the wave travels from line 1 to line 2 , the refractive index at point 1 is $\alpha_{1}$, and the reflective coefficient is $\beta_{1}{ }^{\prime}$; the refractive coefficient at point 2 is $\alpha_{2}$, and the reflective coefficient is $\beta_{2}$. While the wave travels from line 2 to line 1 , the refractive coefficient at point 1 is $\alpha_{1}{ }^{\prime}$, and the reflective coefficient is $\beta_{1}$.

$$
\begin{array}{lll}
\alpha_{1}=\frac{2 z_{l}}{z_{1}+z_{l}}, & \alpha_{1}^{\prime}=\frac{2 z_{1}}{z_{1}+z_{l}}, & \alpha_{2}=\frac{2 z_{2}}{z_{2}+z_{l}} . \\
\beta_{1}=\frac{z_{1}-z_{l}}{z_{1}+z_{l}}, & \beta_{1}^{\prime}=\frac{z_{l}-z_{1}}{z_{1}+z_{l}}, & \beta_{2}=\frac{z_{2}-z_{l}}{z_{2}+z_{l}}
\end{array}
$$

The multiple refraction and reflection between point 1 and point 2 of invasion wave $U_{0}$ could be represented by traveling wave grid graph. Assuming the reflection wave in line 2 has not yet reached point 2, the voltage of point 1 is the superposition of all the refraction and reflection wave at point 1 . The round-trip traveling time of wave in the middle of the lines is $\tau=2 l / v$. Setting the starting time as 
the moment the invading waves first reach point 1 , after time of $t$ (if there is $n$ times refraction and reflection at point 1$)$, the voltage of point 1 is

$$
\begin{aligned}
u_{1}(t)= & \alpha_{1} U_{0}(t)+\alpha_{1} \alpha_{1}^{\prime} \beta_{2} U_{0}(t-\tau)+\alpha_{1} \alpha_{1}^{\prime} \beta_{1} \beta_{2}^{2} U_{0}(t-2 \tau)+\cdots+ \\
& \alpha_{1} \alpha_{1}^{\prime} \beta_{1}^{k-1} \beta_{2}^{k} U_{0}(t-k \tau)+\cdots+\alpha_{1} \alpha_{1}^{\prime} \beta_{1}^{n-2} \beta_{2}^{n-1} U_{0}[(t-(n-1) \tau)]
\end{aligned} .
$$

In the above formulas, $\alpha_{1} \alpha_{1}^{\prime} \beta_{1}^{k-1} \beta_{2}^{k} U_{0}(t-k \tau)$ represents the refraction wave after time of $k \tau$. If $t \geq k \tau$, the amplitude of the refraction wave is $\alpha_{1} \alpha_{1}^{\prime} \beta_{1}^{k-1} \beta_{2}^{k} U_{0}$, while $t<k \tau$, 0 . When $n \rightarrow \infty$, the amplitude of $u_{1}(t)$ is

$$
\left.U_{1}\right|_{n \rightarrow \infty}=\alpha_{1} U_{0}\left[1+\alpha_{1}^{\prime} \beta_{2}\left(1+\beta_{1} \beta_{2}+\beta_{1}^{2} \beta_{2}^{2}+\ldots\right)\right]=\frac{2 z_{l}\left(z_{1}+2 z_{2}-z_{l}\right)}{\left(z_{1}+z_{l}\right)\left(z_{1}+z_{2}\right)} \text {. }
$$

Because the wave impedance of windings is far bigger than the lines', considering the worst case, if we take the transformers as open circuit, total reflection will occur. Then, $z_{1}=350 \Omega, z_{2} \rightarrow \infty, z_{l}=50 \Omega$, and $\alpha_{1}=0.25, \beta_{1}=0.75, \quad \alpha_{1}^{\prime}=1.75, \quad \beta_{1}^{\prime}=-0.75, \quad \alpha_{2}=2, \beta_{2}=1, U_{1} \approx 2 U_{0}$. We can have waveform of $u_{1}$ as shown in fig. 3.

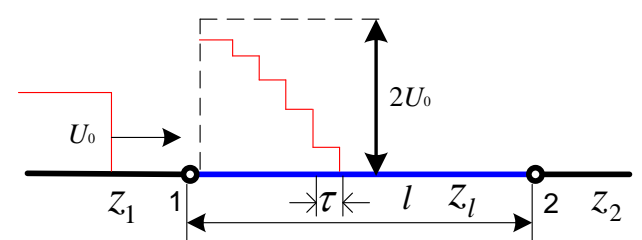

Fig.3 Affection of the line $\mathrm{z}_{l}$ to the invading wave

As we can see, the function of the cables is equivalent to parallel capacitance, can make the gradients of the invading wave flattens. As for the random invading wave, we can use convolution integral or simulation software to solve the problem.

\section{Verification Based on EMTP Simulation}

System Description and Modeling. Assuming that lightning struck one of the overhead ground wires at about $120 \mathrm{~m}$ from the distribution transformer II, flashover accident of A phase occur. The typical 35kV overhead transmission line with double overhead ground wires is as shown in fig. 4.

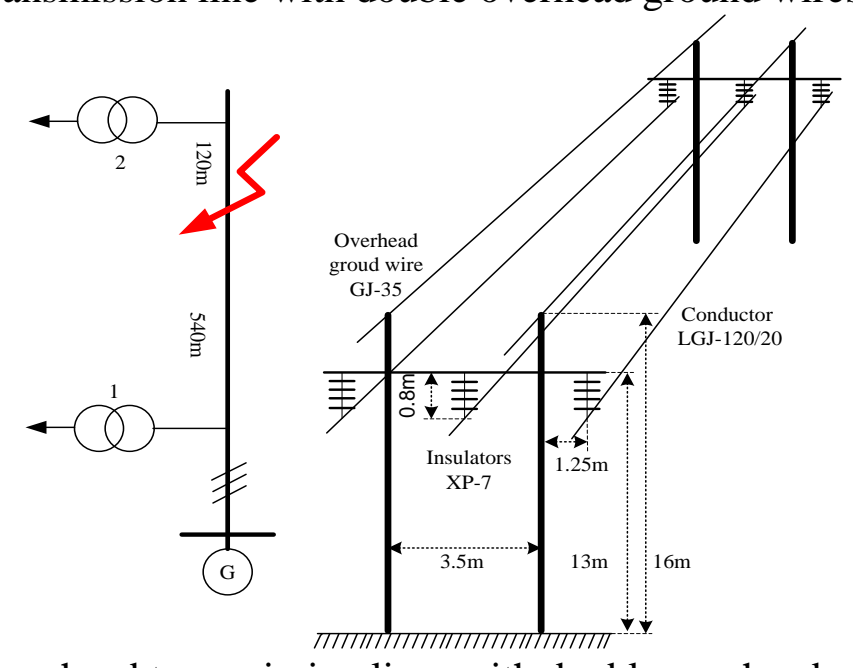

Fig4 35kV overhead transmission lines with double overhead ground wires

Source: Lightning impulse current is modeled using a standard double exponential wave with parameters of $1.2 / 50 \mu$ s, the amplitude is $65 \mathrm{kA}$.

Overhead Transmission Lines: The $\mathrm{OH}$ lines are represented by multi-phase models considering the distributed nature of the line parameters due to the range of frequencies involved. So, we are using JMarti line model with 5 phases.

Resistance of tower and foot tower: The impedance of each reinforced concrete column is about $250 \Omega$, excluding resistive losses. Power frequency grounding resistance value of is approximately $9.8 \Omega$, by using the R-L branches, which consist of resistance of $13 \Omega$ and inductance of $0.005 \mathrm{mH}$, in parallel with the resistance of $40 \Omega$. 
Flashover model: Using the included flash.sup model. Flashover will occur when the voltage between the two ends of insulator strings is over $350 \mathrm{kV}$.

Surge arrester: The surges are the gapless MOV rated at $51 \mathrm{kV}$ with a maximum continuous operating voltage (MCOV) of $40.8 \mathrm{kV}$. The surge arresters are modelled by nolinear resistors.

Transformers: Transformers are represented by capacitance (that is the so-called entrance capacitance) to ground, and the capacitance is $750 \mathrm{pF}$ for each phase.

Cables: The cable is modelled as LCC JMarti model ${ }^{[5-6]}$. It is the most advanced time domain model available as it represents the full frequency dependence of all line parameters. Bare conductors: The impedance of each bare conductor is about $300 \Omega$, excluding resistive losses.

Different connecting wires. The following three cases are simulated and analyzed: 1 . The connecting wire between surge arresters and transformers is $10 \mathrm{~m}$ long bare conductor; 2 . The connecting wire between surge arresters and transformers is $20 \mathrm{~m}$ long single core cable; 3 . The connecting wire between surge arresters and transformers is $20 \mathrm{~m}$ long three-core cable. Assuming that the heights of both single core cables and three-core cables from the ground are $10 \mathrm{~m}$, the power frequency resistance of grounding body of towers is about $10 \Omega$. The simulation model of the entire circuit for the third case is as shown in fig. 5.

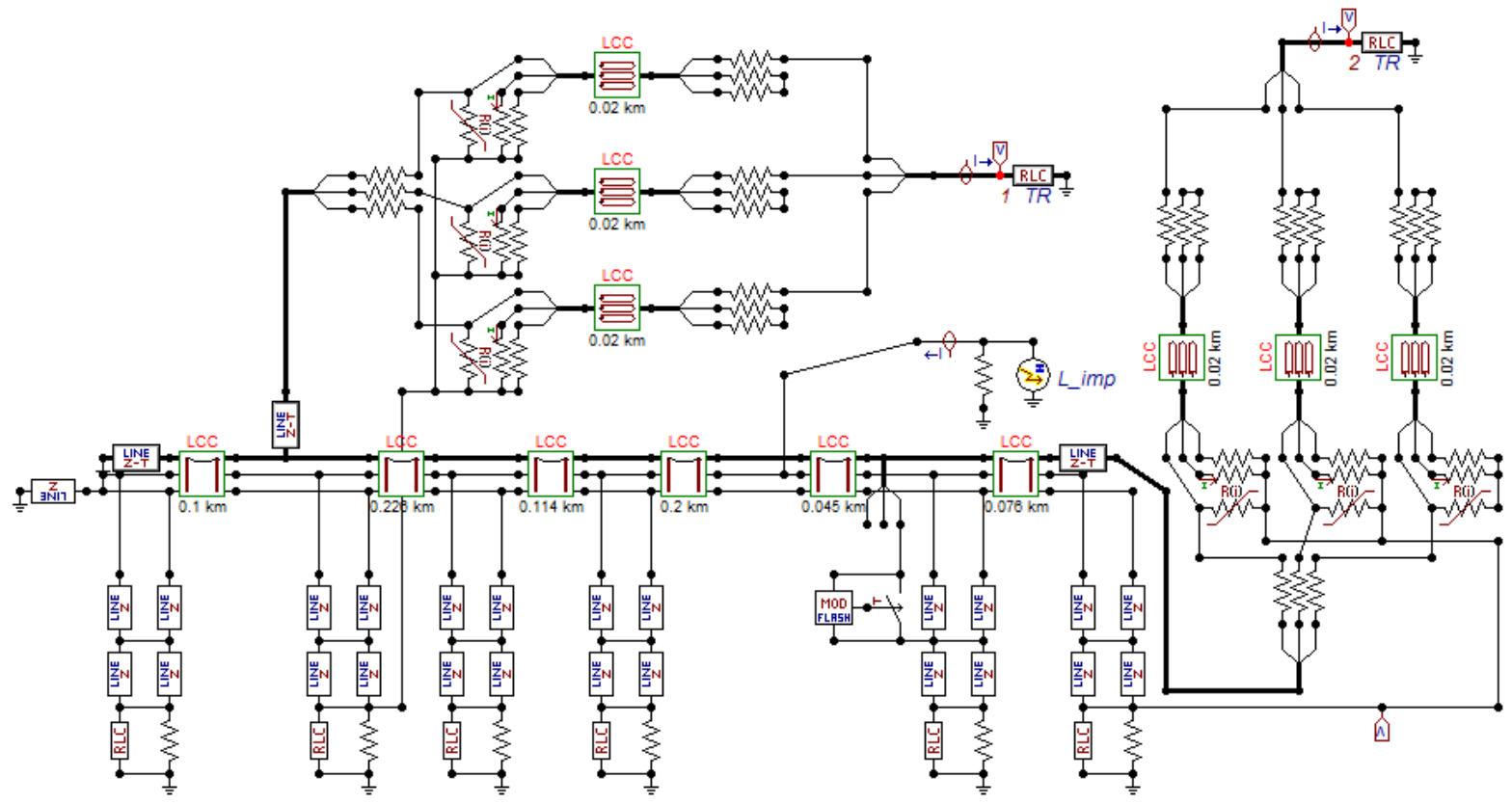

Fig.5 Model of ATPDraw simulation circuit
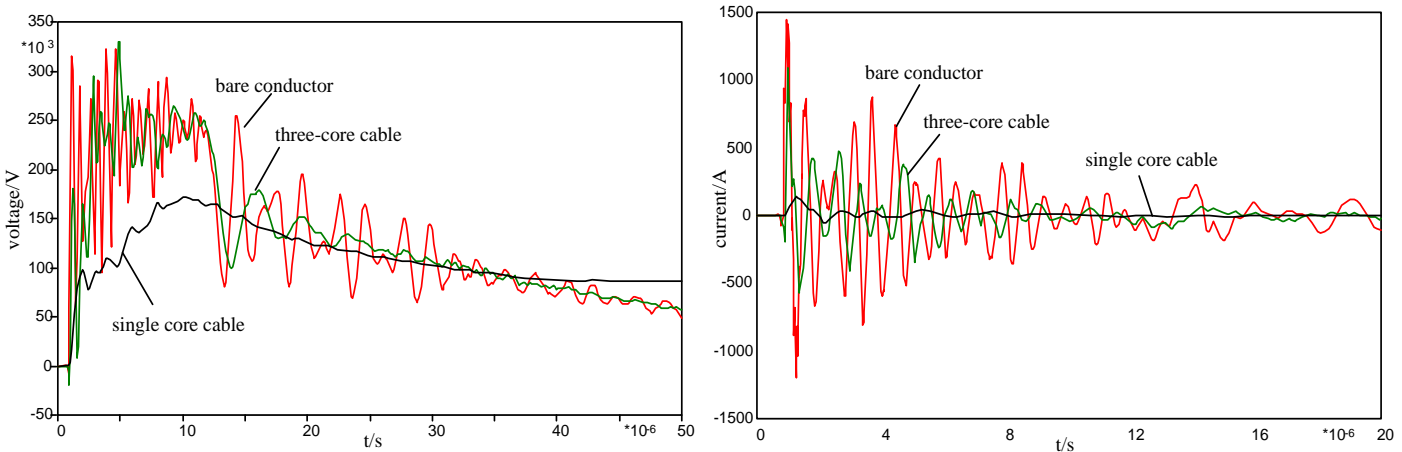

Fig.6 Waveforms of voltage and current when using diferent conductors

Fig6 shows the waveforms of voltage and current of A phase winding inlet terminal at $35 \mathrm{kV}$ high voltage side for number 2 transformer. As for the first case, the amplitude and gradient of the voltage are extremely high. Its amplitude is up to about $320 \mathrm{kV}$, exceeding the lightning insulation withstand strength (about 220kV) of the transformer; and the gradient is extremely high, the transformers will be damaged in an instant. As for the second case, the gradient of the waveform is somehow smaller with respect to the first case, but its amplitude is still a threat to the security of the transformer. And 
for the third case, both the amplitude and gradient are regularly smaller, its amplitude is about $176 \mathrm{kV}$, the transformers can withstand this invasion wave safely.

The currents decay rapidly to zero under all three cases. As for the first case, the amplitude of the current is very high, up to about 1350A, much bigger than the rated current of the transformer's primary windings, which is only about 26A. Although the transient process is very short, the windings of the transformer will suffer from a great electromagnetic force due to the huge inrush current. Because the electromagnetic force is proportional to the square of the current, the electromagnetic force will be hundreds or even thousands times of the rated current when struck by lightning, the huge transient electromagnetic force will make the windings bend and even pull off.

Besides, the huge current will make the windings fever sharply, which is very destructive to its windings and the insulation. Especially for high-voltage motors, the wires subjected to vibration and electromagnetic force will be worn easily for the electric field distribution in the wire slot is extremely uneven.

The gradients of current waveform in the second case is somehow smaller with respect to the first case, but its amplitude is still a threat to the security of the transformer. Under the third case, the amplitude of the current is much smaller, about 140A, which has much smaller impact to the windings.

Different spatial positions. Assuming that the connecting wires all are $20 \mathrm{~m}$ long single core cables, locating at $10 \mathrm{~m}$ high from the ground, surface, and $1.5 \mathrm{~m}$ deep underground respectively. The power frequency resistance of grounding body of towers is about $10 \Omega$. The corresponding waveforms of voltage and current are as shown in fig. 7. In all three cases, the amplitudes of are all less than $220 \mathrm{kV}$. When the cables are placed at ground, the amplitude is bigger than the other two cases. However, the gradient of voltage for overhead cables is bigger than the other two cases, would endanger the vertical insulation of transformer windings. As for the second and third case, the amplitudes of the current are both less than 20A. While in the first case, it is up to 140A.

In conclusion, when the cables are placed on the ground the effectiveness of lightning protection is better than overhead, and underground is better than surface. When cables were placed underground the amplitud and gradients are smaller, within the acceptable range, can protect the transformers effectively.
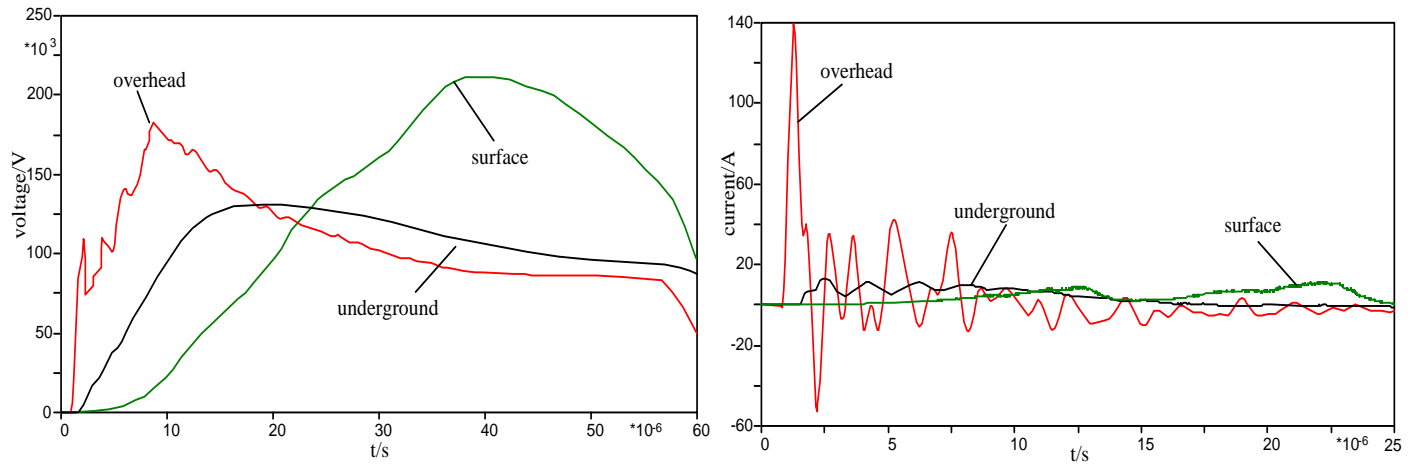

Fig.7 Waveforms of voltage and current when cables are in different spatial positions

\section{Summary}

1) Compared with the bare conductors and three-core cables, single core cables are the best choice for the lightning protection. The length of the cables is better to take $15 \sim 50 \mathrm{~m}$, exceeding $100 \mathrm{~m}$ would be improper.

2) Under the condition that cables are in the same length, the underground cables have better effectiveness than the ground cables and the ground cables are better than the overhead.

Further research will be carried on for circumstances of different grounding resistances and different lengths, so that we can have a more comprehensive thorough protection method. 


\section{References}

[1] P. U. Bakhru, K. E. Bow, D. Fischer, and E. Schrom. Crushing metallic shielded telecommunication cables with dynamic magnetic fields[C]// [S.l.]: [s.n.], 1985: 173-186.

[2] Kenneth E. Bow, Donald A. Volt. Overall shield protects instrument cable from the effects of lightning[J]. IEEE Transactions on Industry Applications, 1994, 30(2): 269-276.

[3] Valsalal P, Usa S, and Udayakumar K. Importance of capacitance on metal oxide arrester block model for VFTO aplications[J]. IEEE Transactions on Power Delivery, 2011, 26(2): 1294-1295.

[4] Morched A, Gustavsen B, Tartibi M. A universal model for accurate calculation of electromagnetic transients on overhead lines and underground cables[J]. IEEE Transactions on Power Delivery, 1999, 14(3): 1032-1038.

[5] Scott-Meyer. EMTP rule book[M]. Manitoba, Canada: Manitoba Research Center, 2003.

[6] Marti J R. Accurate modeling of frequency-dependent transmission lines in electromagnetic transient simulations[J]. IEEE Transactions on Power Apparatus and Systems, 1982, PAS-101(1), 147-157. 